\title{
What do centrality measures measure in psychological networks?
}

\section{Journal Article}

\section{Author(s):}

Bringmann, Laura F.; Elmer, Timon; Epskamp, Sacha; Krause, Robert W.; Schoch, David; Wichers, Marieke; Wigman, Johanna T.W.; Snippe, Evelien

\section{Publication date:}

2019

\section{Permanent link:}

https://doi.org/10.3929/ethz-b-000368126

\section{Rights / license:}

In Copyright - Non-Commercial Use Permitted

\section{Originally published in:}

The Journal of Abnormal Psychology, https://doi.org/10.1037/abn0000446 


\section{What do centrality measures measure in psychological networks?}

Laura F. Bringmann ${ }^{1,6}$, Timon Elmer ${ }^{2}$, Sacha Epskamp ${ }^{3}$, Robert W. Krause ${ }^{4}$, David Schoch $^{5}$, Marieke Wichers ${ }^{6}$, Johanna Wigman ${ }^{6}$, Evelien Snippe ${ }^{6}$

${ }^{1}$ Department of Psychometrics and Statistics, Heymans Institute, University of Groningen, Netherlands

${ }^{2}$ Chair of Social Networks, Department of Humanities, Social and Political Sciences, ETH Zürich, Switzerland

${ }^{3}$ Department of Psychological Methods, University of Amsterdam, Netherlands

${ }^{4}$ Department of Sociology/ICS, University of Groningen, Netherlands

${ }^{5}$ Department of Sociology, University of Manchester, UK

${ }^{6}$ Interdisciplinary Center Psychopathology and Emotion Regulation (ICPE), Department of

Psychiatry (UCP), University of Groningen, University Medical Center Groningen,

Netherlands

Address correspondence to:

Laura Bringmann, $\mathrm{PhD}$

Department of Psychometrics and Statistics

Grote Kruisstraat 2/1

University of Groningen

9712 TS Groningen

Netherlands

Email: 1.f.bringmann@rug.nl

Phone: +31503639737

Conflict of Interest: The authors declare that they have no conflicts of interest.

Funding: Johanna Wigman was supported by the Netherlands Organization for Scientific Research (NWO; Veni grant no. 016.156.019). Sacha Epskamp was supported by Netherlands Organization for Scientific Research (NWO; Veni grant no. 016.195.261). Evelien Snippe was supported by the Netherlands Organisation for Health Research and Development (ZonMw, Off Road grant no. 451001029). Marieke Wichers received funding from the European Research Council (ERC) under the European Union's Horizon 2020 research and innovation programme (ERC-CoG-2015; No 681466 to M. Wichers.

Acknowledgements: We would like to thank Markus Eronen, Kieran Mepham, Denny Borsboom, and Marijtje van Duijn for important comments and discussions on earlier drafts of this paper. 


\begin{abstract}
Centrality indices are a popular tool to analyze structural aspects of psychological networks. As centrality indices were originally developed in the context of social networks, it is unclear to what extent these indices are suitable in a psychological network context. In this paper we critically examine several issues with the use of the most popular centrality indices in psychological networks: degree, betweenness, and closeness centrality. We show that problems with centrality indices discussed in the social network literature also apply to the psychological networks. Assumptions underlying centrality indices, such as presence of a flow and shortest paths, may not correspond with a general theory of how psychological variables relate to one another. Furthermore, the assumptions of node distinctiveness and node exchangeability may not hold in psychological networks. We conclude that, for psychological networks, betweenness and closeness centrality seem especially unsuitable as measures of node importance. We therefore suggest three ways forward: (1) using centrality measures that are tailored to the psychological network context, (2) reconsidering existing measures of importance used in statistical models underlying psychological networks, and (3) discarding the concept of node centrality entirely. Foremost, we argue that one has to make explicit what one means when one states that a node is central, and what assumptions the centrality measure of choice entails, to make sure that there is a match between the process under study and the centrality measure that is used.
\end{abstract}

Keywords: Centrality; Psychopathology; Psychological networks; Social networks; Network analysis. 
Running head: WHAT DO CENTRALITY MEASURES MEASURE?

General summary: In clinical psychology, networks of symptoms or affect states are increasingly used to study psychopathology. Such psychopathological networks are often further analyzed with centrality measures that indicate which symptoms or affect states are structurally important. We argue that the use of these centrality measures, which originally stem from social networks, is problematic in psychological networks, and propose several alternative ways forward. 


\section{Introduction}

Networks or graphs are a general way to visualize and analyze the interaction between nodes. The most well-known networks are social networks, which have been used and studied for decades (Newman, 2010). In a social network the nodes are people (or groups of people) and these nodes are connected through some sort of relation, such as friendship. One could study, for example, if schoolchildren are more likely to be friends with schoolmates of the same gender (Moreno, 1934; Newman, 2010). In network studies, the emphasis is thus on the connections between the nodes of the network (Kolaczyk \& Csárdi, 2014).

Recently a new kind of network has been introduced: the psychological network (Borsboom, 2008; Borsboom \& Cramer, 2013; Cramer, Waldorp, van der Maas, \& Borsboom, 2010). Such psychological networks are different from social networks, as the nodes in the network are not people but psychological variables, such as affect states or symptoms (Cramer et al., 2012; Fried \& Cramer, 2017; Fried et al., 2017; Klippel et al., 2017; van Roekel, Heininga, Vrijen, Snippe, \& Oldehinkel, 2018). In psychological networks, the nodes are operationalized as, for example, items of a depression questionnaire such as the Beck Depression Inventory (Bringmann, Lemmens, Huibers, Borsboom, \& Tuerlinckx, 2015; David, Marshall, Evanovich, \& Mumma, 2018).

Another difference is that in social networks the connections between nodes are considered to be observable, that is, they feature as data. People self-report on a certain relation of interest, or it is reported by an external observer (e.g., whether person A is friends with person B). A social network, thus, can be seen as a reflection of these raw data. In psychological networks, however, the connections between the nodes (e.g., symptoms) are not treated as data $^{1}$, but as parameters that have to be inferred using existing modeling techniques. The most

\footnotetext{
${ }^{1}$ In the present paper, we focus exclusively on networks based on statistical parameters, which are currently popular; networks in which people are asked to rate the relations between symptoms directly (e.g., Frewen,
} 
popular models to estimate a psychological network are partial correlations for cross-sectional data (i.e., cross-sectional network; Costantini et al., 2015; Epskamp, Waldorp, Mõttus, \& Borsboom, 2018; van Borkulo et al., 2014) and vector autoregressive based models for intensive longitudinal data (i.e., temporal network; Bringmann et al., 2013). Thus, instead of being direct representations of raw data, the connections in psychological networks represent coefficients derived from a model, such as partial correlation coefficients or regression weights.

Especially in mental health research, the application of psychological or more specifically psychopathological networks has drastically increased in the past five years. In psychopathological networks, the focus is on individual symptoms or psychological states (measured at a smaller scale) and how such symptoms influence each other. For example, sleeping problems can lead to tiredness, which in turn can trigger sadness, and as the downward spiral progresses, symptoms reinforce one other, eventually resulting in a full-blown depression (Borsboom, 2017; Cramer et al., 2010). Symptom networks have been inferred and analyzed for several psychopathological domains, including depression (Boschloo, Schoevers, van Borkulo, Borsboom, \& Oldehinkel, 2016; Boschloo et al., 2015; van Borkulo et al., 2015), psychosis (Isvoranu, Borsboom, van Os, \& Guloksuz, 2016; Isvoranu, van Borkulo, et al., 2016; Wigman, de Vos, Wichers, van Os, \& Bartels-Velthuis, 2016), and posttraumatic stress disorder (McNally et al., 2015). Besides permeating research, network analytic tools are currently also being explored in clinical practice in the form of network-based interventions (Bak, Drukker, Hasmi, \& van Os, 2016; Kroeze et al., 2017). This approach is appealing not only due to the plausibility of the idea that to understand psychopathology one should focus on the elements constituting this process - the interplay between symptoms and external factors

\footnotetext{
Allen, Lanius, \& Neufeld, 2012; Ruzzano, Borsboom, \& Geurts, 2015) and for networks constructed on the basis of diagnostic systems (Borsboom, Cramer, Schmittmann, Epskamp, \& Waldorp, 2011; Tio, Epskamp, Noordhof, $\&$ Borsboom, 2016) require a separate analysis.
} 
(Fried \& Nesse, 2015; Wichers, 2014) - but also due to the useful visualization tools (Epskamp, Cramer, Waldorp, Schmittmann, \& Borsboom, 2012), that depict partial correlation and vector autoregressive models as a network in an intuitive way (Bringmann \& Eronen, 2018).

In addition to the visualization of networks, the network approach to mental disorders leads to a whole new toolbox to analyze the interrelations of symptoms that originally stems from social network analysis. This toolbox includes centrality indices, of which the most commonly used are degree, closeness, and betweenness centrality. Centrality indices are intended to reveal the relative importance of nodes in the structure of the network. Symptoms with a high centrality may be the ones that strongly affect other symptoms in the network due to their strong connections to other symptoms (Borsboom et al., 2011; Newman, 2004). The identification of such potentially influential symptoms is thought to be of importance because it could guide the choice of symptoms to intervene on in clinical practice (Borsboom \& Cramer, 2013). Corresponding to these suggestions, centrality indices have been used in empirical papers to describe the importance of symptoms within the network structure, including several recent articles in the Journal of Abnormal Psychology (Anker et al., 2017; Goldschmidt et al., 2018; Levinson et al., 2017; Moshier et al., 2018; Preszler, Marcus, Edens, \& McDermott, 2018; Robinaugh, Millner, \& McNally, 2016; Verschuere et al., 2018).

However, recently several researchers have also raised doubts about the use of centrality indices in psychological networks (Bringmann, 2016; Epskamp, 2017; Epskamp, Borsboom, \& Fried, 2017; Epskamp, van Borkulo, et al., 2018). First, these centrality indices were originally developed for social networks, which, as we have seen, differ from psychological networks in important ways. Moreover, even in the social network context for which they were developed centrality indices have been far from unproblematic in terms of interpretation and conceptualization (Borgatti, 2005; Freeman, 1979). This casts doubt on the suitability of these indices for analyzing psychological or psychopathological networks. 
Second, centrality indices, especially closeness and betweenness centrality, have been shown to be unstable in some cross-sectional and temporal networks (Bulteel, Tuerlinckx, Brose, \& Ceulemans, 2016; Epskamp et al., 2017). Centrality indices have, for instance, been observed to display wide confidence intervals (for betweenness centrality; Bringmann et al., 2013), low stability in cross-sectional data (Epskamp et al., 2017), or inconsistency in findings regarding the most central node across datasets of similar psychological variables (Bringmann et al., 2016; Forbes, Wright, Markon, \& Krueger, 2017, however, see also Borsboom et al., 2017).

Third, in addition to the reliability concerns, little research has been done on the predictive power of centrality indices. Research using cross-sectional networks has found some evidence for the idea that central symptoms more strongly predict the onset of major depressive disorder than less central symptoms (Boschloo, van Borkulo, Borsboom, \& Schoevers, 2016). However, a study on social anxiety disorder (Rodebaugh et al. 2018) found that although degree centrality (not closeness or betweenness centrality) seemed to have some utility in predicting change processes and social anxiety severity, it was simply the number of times that patients endorsed (i.e., reported) a symptom that had the most predictive power in indicating which items were the most important ones. The authors conclude that clinicians could use highly central symptoms of cross-sectional networks, but simply treating the most commonly reported symptoms would probably work better (Rodebaugh et al., 2018). Furthermore, there remains a lack of studies on the predictive value of centrality indices in temporal networks.

Thus, even though centrality indices seem intuitive, easily applicable and are often used (Boschloo, Schoevers, et al., 2016; Fried, Epskamp, Nesse, Tuerlinckx, \& Borsboom, 2016; Marcus, Preszler, \& Zeigler-Hill, 2018; Robinaugh, LeBlanc, Vuletich, \& McNally, 2014), possible issues in interpreting these indices in the context of psychological networks have not yet been reviewed. In this paper, we will critically examine which issues have been raised in 
the social network literature and to what extent these issues transfer to psychological networks. To this end, we will consider, from a conceptual point of view, the three most used network measures in the psychological literature: degree, closeness, and betweenness centrality.

The structure of the paper will be as follows: We will first go through the general definitions and explanations of the three centrality indices based on the social network literature. In the next section, we will dive into the known problems these measures have in social networks and discuss these and other issues in applying them to psychological networks. In the final section, we will suggest several ways to move the field of psychological networks research forward.

\section{Centrality: The Background}

The centrality indices used in psychological networks originally stem from the field of social networks (Newman, 2010) and were originally developed in the context of human communication (Bavelas, 1950; Leavitt, 1951). In social networks, for example friendship networks, one studies the relationship between social entities called actors. Actors are discrete separable entities or nodes, for example, individuals or companies. The relationships between these nodes are called edges, links, or ties and can range from evaluations of one person by another (friendship networks) to transfer of material resources (transaction networks). These relations can be gathered through, for example, questionnaires, interviews, observations, or databases (e.g., for citation networks where the nodes are researchers; Wasserman \& Faust, 1994). Such relations can be captured in an adjacency matrix, in which an entry equals one if there exists an edge between node $i$ and node $j$, and is zero otherwise. The adjacency matrix can then be used to represent the network as a graph. However, for simplicity we will only use the word network in this paper. Besides visualization of such relations, networks are commonly analyzed with statistical tools to examine structural properties. These range from macro level 
features (e.g., the small world effect; Borsboom et al., 2011; Watts \& Strogatz, 1998) through meso level features (e.g., clustering; Fortunato, 2010) to micro or node level features of the network (Wasserman \& Faust, 1994). The latter includes applications of centrality indices to identify structurally important nodes within the network.

Even though centrality is one of the key concepts in social network analysis, there was historically (and still is) no generally accepted conceptualization for its measurement, due to the ambiguity of being structurally important (Freeman, 1979). This led to a great variety of possible measures of centrality. Additionally, most measures were so complex that it was unclear what they were supposed to measure (Freeman, 1979). In order to bring some clarity to this strand of research, Freeman tried to reevaluate the concept of centrality and measures that had been introduced, and distilled from that three centrality indices: degree, betweenness, and closeness. These became very popular measures of centrality for unweighted networks, in which edges have only two possible values, one (an edge) or zero (no edge), and still form the basis of many centrality indices (nowadays there are over 100 centrality measures to choose from; Lü et al.,2016)2 ${ }^{2}$, including the ones we will discuss below (Wasserman \& Faust, 1994). More specifically, we will focus on Opsahl's centrality indices for weighted networks (i.e., the edges have a weight assigned to them; Opsahl, Agneessens, \& Skvoretz, 2010), which are adapted from Freeman's centrality indices. These three centrality indices are implemented in the $R$ package qgraph (Epskamp et al., 2012) and are by far the most commonly used to analyze psychological networks.

\section{Degree and Strength Centrality}

\footnotetext{
${ }^{2}$ See also: http://schochastics.net/sna/periodic.html
} 
Degree centrality is simply the sum of direct (i.e., adjacent) edges each node has (Freeman, 1979; Wasserman \& Faust, 1994). To illustrate the calculation of degree centrality, we show in Figure 1 an example of a possible psychological network of 9 nodes that are connected through 9 (weighted) edges. Such a network could represent, for instance, partial correlations (edges) connecting psychological variables, such as symptoms (nodes). In this network, node 3 has the most edges directly connected to it and thus with a degree of 4 , with edges between the nodes $(3,1),(3,2),(3,4)$, and $(3,5)$, has the highest degree centrality. The concept of degree centrality is most easily explained in its original social networks context, for example, a network of schoolchildren playing together outside of the classroom. The network can be constructed by observing the children (the nodes) and coding an edge when two children play together. The children with a low centrality degree would be the ones who do not play with many others, whereas a child with a high degree centrality would be a child that does play with many other children. In psychopathological terms, the node with the highest degree is a symptom that directly interacts or is associated with many other nodes or symptoms in the network (Richetin, Preti, Costantini, \& De Panfilis, 2017).

As Figure 1 is a weighted network, one could also, instead of just counting if there is an edge or not, take the edge weights into account, which is known as strength centrality (Barrat, Barthelemy, Pastor-Satorras, \& Vespignani, 2004; Newman, 2004). Psychological networks usually contain positive and negative edge weights, as in the current example, whereas strength centrality was originally defined for networks with only positive edge weights. Thus, in psychological networks strength centrality is calculated by taking the sum of all absolute edge weights a node is directly connected to. In this case, node 3 again has the highest strength centrality $(0.27+0.45+0.16+0.11=0.99)$. Furthermore, besides being undirected, edges can also be directed, for example, when using vector autoregressive models. In directed networks, there is thus an indegree or instrength centrality and an outdegree or 
outstrength centrality: nodes that receive the most edges and nodes that send the most edges (Wasserman \& Faust, 1994). ${ }^{3}$ This is a useful distinction, as such directed network models are thought to provide information both on how a symptom directly activates and is activated by other symptoms (Fried et al., 2016).

\section{Closeness Centrality}

At the core of network science is the interest in how edges connect nodes across the whole network. For studying this, the concept of path length is used: A path length is the number of steps (edges) it takes to get from one node to another (Scott, 2000). A limitation of degree (and strength) centrality is that only direct ties or paths of length 1 are taken into account. However, often nodes in a network are indirectly connected, with a path length of 2 or more. It has been argued that these indirect connections should also be taken into account, and thus a global measure of centrality is needed (Scott, 2000). One such global centrality measure is closeness. The basic idea is simple: an individual or node has high closeness centrality if the information from this node can reach other nodes quickly (Wasserman \& Faust, 1994), and the node can thereby, for example, communicate in an optimal or efficient way with all other individuals (or nodes) in the network and get to resources quickly (Freeman, 1979). In psychopathological networks, it is usually thought that a symptom with high closeness centrality is more likely to quickly affect other symptoms, and changes in other symptoms are more likely to affect symptoms with high closeness centrality (Rhemtulla et al., 2016; Richetin et al., 2017; Smith, Lee, Martel, \& Axelrad, 2017). A plausible way to quantify closeness or minimum reachability time is by finding the fastest route between two nodes (often referred to

\footnotetext{
${ }^{3}$ The most common weighted directed networks in psychology are based on vector autoregressive models, in which case there can also be edges going back to the node itself, so called self-loops. These self-loops are normally not taken into account when calculating centrality indices.
} 
as the geodesic distance; Sabidussi, 1966). The fastest route for an unweighted network can be calculated by defining the shortest paths. Looking back at the network in Figure 1 without taking the edge weights into account, the shortest path, for instance from node 5 to node 1 , is via node $3(5,3,1)$ as it has a path length of two, whereas via node $4(5,4,3,1)$ it has a path length of three.

Because Figure 1 and most typical psychological networks (e.g., based on partial correlations or vector autoregressive coefficients) are weighted networks, when calculating closeness centrality, it is important to take not just binary paths (path or no path) into account, but also the connection strengths. For instance, consider the nodes 5 and 3 . There is a direct connection between these nodes, which we could interpret as the shortest path. However, the indirect path $5-4-3$ features stronger edge weights. It may therefore be more likely that information spreads from 5 to 3 via 4 rather than via the weak direct path. Notice that a high (again, absolute) edge weight indicates a faster connection between two nodes. To derive a measure for distance between two nodes, we take the inverse of the edge weight (i.e., 1/|weight|). With this information, we can calculate the geodesic distance/shortest path between two nodes.

To continue the example of calculating closeness for node 5, the weighted path length from nodes 5 to 1 via node 3 is 12.8 (node 5 to 3 is $1 / 0.11=9.1$ and node 3 to 1 is $1 / 0.27=3.7$, summed together 12.8), whereas via node 4 the weighted path length is 12.45 (node 5 to 4 is $1 / 0.4=2.5$, node 4 to 3 is $1 / 0.16=6.25$ and from node 3 to 1 gives again $1 / 0.27=3.7$, summed together 12.45). This indeed confirms that the fastest route from node 5 to 1 is via node 4 instead of 3 once the edge weights are taken into account. In the same way, the shortest path lengths are found from node 5 to every other node in the network. To obtain the closeness measure for node 5, these calculated shortest paths are then summed together, resulting in 141 . As this sum score is actually a measure of distance, how far away a node is from all others, we 
again have to take the inverse of the summed shortest path length for each node to calculate closeness centrality. For node 5 this is $1 / 141=0.007$, which happens to be one of the highest closeness centrality values (see the right panel of Figure 1).

In temporal psychopathological networks, closeness centrality has been described and interpreted in the same way as in cross-sectional networks. For example, a mood variable with high closeness centrality is seen as being close to other mood variables and thus able to interact with them quickly (Bringmann et al., 2016; Wigman et al., 2015). What has been overlooked in the context of directed psychological networks, however, is that the shortest path length can differ going from one (from node 5 to node 1) or the other direction (from node 1 to node 5). In this case, the matrix containing the shortest path values can be asymmetric, leading to a measure of in-closeness and out-closeness centrality, just as with strength centrality (Scott, 2000, p. 86). In other words, in-closeness takes all the incoming edges of nodes into account when computing the shortest path, and out-closeness all the outgoing ties. Notice that in the $R$ package qgraph (up till version 1.5) only the out-closeness centrality will be calculated in the case of directed networks.

\section{Betweenness Centrality}

Another global measure defined by Freeman is betweenness centrality. Betweenness centrality, independently introduced by Anthonisse (1971) and Freeman (1977), quantifies the relative number of shortest paths passing through a specific node. A node with high betweenness can influence the information flow between non-adjacent (not directly connected) nodes (e.g., individuals), and thus has an important intermediary or gatekeeper position. In psychopathological networks, a symptom with high betweenness centrality is described as one that lies along the shortest paths between two other symptoms and is able to funnel the flow in the symptom network (Bringmann et al., 2013; Rhemtulla et al., 2016). 
Freeman and others suggested in the 1970's that betweenness centrality can be defined by calculating how often a node is on the fastest route between two other nodes (Wasserman $\&$ Faust, 1994). The fastest route can be the shortest path (unweighted network) or the shortest path length (weighted network). As we have seen in the previous section, shortest path and path length are not necessarily the same and thus betweenness centrality can also be different depending on whether the edges are weighted or not. Take as an example node 4 in Figure 1. If the network were unweighted, this node would have a betweenness centrality of 0 as it is never on a shortest path between other nodes. However, in this weighted network, node 4, due to its high edge weights, is, in contrast, on many shortest paths and has one of the highest betweenness centrality values (see Figure 1 right panel). The same applies to node 6, which has the highest betweenness centrality and thus a gatekeeper role (Scott, 2000). In order to get from node 1 (or $2,3,4,5,7$, or 8 ) to node 7,8 , or 9 the information always has to go through node 6 , resulting in 17 shortest path lengths on which node 6 lies, and thus a betweenness centrality of 17.4

Betweenness centrality takes the directions of the edges into account when calculating shortest paths, which means that results can be different for undirected and directed networks (Gould, 1987; White \& Borgatti, 1994). Because betweenness is a measure of how often a node is intermediate in an information flow between other nodes, and does not take into account how much information a node sends or receives, there is no such thing as in- or out-betweenness.

\footnotetext{
${ }^{4}$ Sometimes the shortest path lengths are doubled, resulting in a betweenness centrality value of 34 for node 6 , as it can be argued that the information flow also goes the other way around. For example, node 6 is on the shortest path from node 1 to node 9 , but also from node 9 to node 1 . However, this does not influence the ranking of the betweenness centrality index. Furthermore, it is relevant to note that if there is not just one but two (or more) shortest paths between node A and B, then betweenness centrality is not based on the absolute number of shortest paths a node lies on, but the relative number. For example, it might happen that there are two equally short paths between $\mathrm{A}$ and $\mathrm{B}$, with a third node $\mathrm{C}$ lying on one of them. Then $\mathrm{C}$ has $1 / 2$ added to its betweenness centrality and not 1 . If the absolute number instead of the relative number of shortest paths is taken, then we are using stress centrality (Shimbel, 1953).
} 


\section{Issues with Centrality Indices: From Social to Psychological Networks}

After having taken a deeper look at how centrality indices have been developed in social networks and how they are used in psychological networks, in this section we will consider the possible issues with interpreting centrality indices. As is the case with many statistical techniques, inferences based on the three centrality indices rest on (possibly implicit) assumptions, which make them suitable or valid in some contexts but invalid and uninterpretable in other contexts. As an analogy, consider calculating the mean. Although the mean can in principle always be calculated when you have numbers (Lord, 1953), the interpretation of the mean can lead to confusing results when applied to nominal numbers assigned to categorical data, for example numbers assigned to nationalities. In this case, the mean could be 2, indicating that the average person is, say, Finnish, even though there is only one Finnish person in the sample at hand. ${ }^{5}$ Of course, this does not imply that we should never use the mean as a statistical measure. Similarly, whether centrality indices are suitable depends on the extent to which assumptions are satisfied. In this section, we will look into these assumptions, and examine what they entail for both social and psychological networks.

One possible approach to study the suitability of centrality indices is by conceptualizing networks in terms of flow processes (Borgatti, 2005). Borgatti distinguishes between three different flow processes: parallel, serial, and transfer. Whereas parallel and serial flow processes occur via replication or copying, either in parallel or one at a time (i.e., serially), in a transfer flow process things (e.g., money or post) simply move around a network. An example of a parallel flow process is an e-mail network in which people send out e-mails to warn of a

\footnotetext{
${ }^{5}$ Imagine that a student wants to find out what is the most common nationality in a group of 13 students (7 from Germany, 1 from Finland, 3 from the Netherlands, and 2 from the US). During data collection, the student assigns a number to each nationality ( $1=$ German, $2=$ Finnish, 3=Dutch, 4=American). We thank Susan Niessen for this example.
} 
16

Running head: WHAT DO CENTRALITY MEASURES MEASURE?

computer virus. This can be seen as a parallel process, as emails spread through the network simultaneously, every individual sending emails to all their contacts at once. Such parallel processes can be captured with degree (or strength) or closeness centrality. An example of a serial process is a disease, such as human immunodeficiency virus (HIV), which is transferred one individual at the time, spreading further and further through a network, while an individual that has been infected stays infected. According to Borgatti (2005) none of the centrality indices can capture these kinds of flow processes. Finally, an example of transfer flow is the delivery of a package, for instance, a mail carrier delivering the post. The mail carrier aims to pass by the houses via the most efficient route possible to reach her destinations (the different addresses), and once she has delivered a package, she no longer has it herself. This last flow can be described with the betweenness and closeness centrality indices.

Whereas Borgatti (2005) lays out the different flow processes for most social networks, it has not yet been clarified what the process of flow under study in psychological networks is like. It can be argued that, when talking about symptom spread, the process is like a serial flow, where symptoms affect each other one at the time, such as with gossip or HIV. In this case, none of the centrality indices are suitable measures for capturing the flow in psychological networks, as all indices (degree, betweenness, and closeness centrality) are unsuited for serial processes such as the spread of gossip. However, it is perhaps even more plausible that in psychological networks symptom spread happens in a parallel way. A parallel flow, such as email spread, can be modeled with a degree centrality. In this type of flow, one does not need to necessarily know the information flow going through the whole network, instead, looking at path length 1 is enough to find the most influential node (e.g., the person that has the most email contacts). In the case of symptom networks this would mean that symptoms do not affect other symptoms step by step in a serial fashion, but rather affect everything they are directly 
connected to in a parallel way. Which dynamic process best captures the spread of symptoms in a network is thus currently an open question.

Yet, it is questionable whether the idea of flow is meaningful at all in psychological networks. Originally, flow networks were conceptualized as directed networks that described transportation processes, such as traffic or fluids in pipes (Newman, 2010). These are networks where the edges indeed directly represent a flow process. However, even in social networks one can come up with examples where a structure is unlike a flow process. For example, having or not having a lot of friends in a friendship network is difficult to conceptualize as a flow process, as there is nothing literally transferring between people. Similarly, in psychological networks, we might have a conceptual idea on how symptoms spread through the network, but the network model and thus network structure does not automatically reflect this conceptualization. On the contrary, there is nothing flowing between the symptoms. Rather, a cross-sectional network gives information on the strength of predictive associations between affect items or symptoms and temporal networks inform us about how, for instance, the change in one symptom is likely to predict a change in other symptoms at the next time point (i.e., a lag-1 VAR model). These models give information on direct connections but not on how symptoms would affect each other indirectly (in terms of a path length exceeding 1) through the whole network (Epskamp, 2017). They both thus give valuable information on relation strength between nodes, but do not correspond to a flow process in a straightforward way.

Apart from flow processes, Borgatti points out several other assumptions that these centrality indices have (Borgatti, 2005; Borgatti \& Everett, 2006). For instance, closeness centrality comes with the assumption that each node or person is trying to reach all other nodes in the network or trying to get information (such as a virus or gossip) to all other nodes in the network. This can be seen by the fact that it is calculated based on the shortest paths between nodes, taking all the paths between all nodes in the network into account. However, the 
assumption that each node tries to reach all other nodes may not be plausible in some networks, such as networks representing romantic relationships between people. A further drawback of the closeness centrality measure is that when a node is not reachable (there is no path going to it), the distance cannot be calculated and the distance sum will go to infinity (i.e., is undefined), making closeness centrality unsuitable. This means the measure is only applicable to fully connected networks (when all nodes can be reached by the other nodes; Wasserman \& Faust, 1994, p. 203). ${ }^{6}$ These issues transfer to psychological networks as well. For example, the requirement of a fully connected network makes the closeness index unsuitable in many instances, as psychological networks, just as social networks, are often not fully connected (e.g., in the case that the Lasso penalization is used for directed networks; Epskamp et al., 2017).

For most social and psychological networks, an even more problematic assumption required for both betweenness and closeness centrality is the aforementioned assumption of shortest paths. The idea behind this assumption is that if, for example, people communicate with one another, they will do that in the most efficient way and thus will take the shortest route, no matter who the nodes in the network are (Stephenson \& Zelen, 1989). This seems reasonable for a transfer process, such as a package delivery, but one can also imagine many processes that will take a less efficient or more indirect route in the network. This can happen, for example, due to social preferences regarding individuals you want to share information with, as in the case of gossip. Moreover, often the process under study does not even know the shortest way in the network (e.g., viruses, gossip, or money transfer), which makes it even less likely that information will go through the network in the "best" or most efficient way

\footnotetext{
${ }^{6}$ Note that there exist alternative closeness centrality measures in which not all nodes need to be reachable and thus the network need not be fully connected, for example, the integration measure by (Valente \& Foreman, 1998). See also footnote 1 in Opsahl et al. (2010).
} 
(Borgatti, 2005). Therefore, although widely used in social networks, most of the time betweenness and closeness indices are not suitable to detect central nodes at all (Borgatti, 2005). For the same reason, these indices are also unlikely to fit psychological networks if they are used as a basis for dynamic conclusions in the absence of evidence that the dynamics actually respect the structure of the network in the appropriate way. Most importantly, it is unclear what entity in a symptom or affect network would follow a path at all, as these networks are about connection strengths between symptoms and not about transmitting something from one symptom to another. With this in mind, it is questionable whether this idea of distance and thus shortest or most efficient paths is meaningful in psychological networks.

What adds to this conundrum is that in psychological networks the edges are often negative, whereas degree, closeness, and betweenness were developed with distance or path lengths in mind, and length cannot be negative. Taking the absolute value is one option, just like splitting the network into negative and positive edges (and again taking the absolute values). Nonetheless, in both cases important information, namely, that some edges are influencing other symptoms negatively instead of positively, will get lost. Therefore, for instance, degree centrality only indicates how locally influential a node is, but not whether the influence is negative or positive. This makes all indices, even degree centrality, suboptimal for many psychological networks.

What may sometimes also make centrality indices less interpretable in the psychological context than in the social context is the issue of node distinctiveness (Bulteel et al., 2016). In social networks, the nodes are usually clearly distinct: they are different people and thus no overlap between nodes is possible (of course node distinction can become fuzzier in social networks too, for example when the nodes are companies). However, in psychological networks, the opposite is the case. The nodes, as they are typically based on items of a questionnaire, often have some form of multicollinearity and thus have both unique and shared 
variance. The latter is typically not represented in the network edges and thus centrality measures are mostly based on this unique variance, missing information on the shared variance (Bulteel et al., 2016; Robinaugh et al., 2014). Furthermore, one could argue that in order to state that node 1 is more influential than node 2 , they need to be truly distinct entities (Bringmann \& Eronen, 2018; Fried \& Cramer, 2017). Thus, one could argue that if two nodes are not truly distinct but conceptually overlap, it is problematic to claim that one of them is more central than the other.

Another assumption that hampers the use of these centrality indices in psychological networks is node exchangeability (Snijders, 2011). For most centrality indices it is assumed that nodes or people are interchangeable in the computation of the centrality indices (i.e. we count the number of paths without distinguishing between them). This implies that there are no relevant qualitative differences between the nodes in addition to the specific connections they have to other nodes. In psychological networks, especially symptom networks, this seems, however, not to be the case. According to the DSM (American Psychiatric Association, 2013), suicidal thoughts is a qualitatively more severe symptom than, for instance, loss of interest in sex. For this reason, it seems problematic to focus only on the connections in psychological networks to find the most central node (Bringmann, 2016). Instead, to be able to make statements about which symptom is the most important, centrality indices that also take node attributes such as severity of symptoms into account should be considered.

Additionally, the calculation of centrality indices is strongly affected by the set of nodes that make up the network. Every network representation assumes that all relevant nodes for the system under study are included. This ultimately raises the question of network boundaries: which nodes (i.e., variables) should be included in the network? Defining the set of nodes is a crucial decision, and has also been extensively discussed in research on social networks (e.g., see Laumann, Marsden, \& Prensky, 1989). In particular, centrality measures that take into 
account shortest paths (e.g., betweenness centrality) or distances (e.g., closeness centrality) can change drastically when a node is removed from or added to the network (Costenbader \& Valente, 2003, Epskamp et al., 2017).

Lastly, in order to know if a centrality measure indeed describes something being central in a network, it is important to have conceptual clarity on what is meant when one states that a node (e.g., person or symptom) is central, and to have a network structure that fits or approximates this conceptualization (Freeman, 1979). The importance of having a clear conceptualization of centrality can be seen in the context of criminal networks (Firmani, Italiano, \& Laura, 2014). In the research of Duijn, Kashirin, and Sloot (2014), criminal cannabis networks were studied. In these networks, nodes represented criminals and edges represented social contacts between the criminals. Using degree and betweenness centrality, Duijn et al. (2014) tried to find the most important or influential criminals. Degree was in this case interpreted as having access to information or resources and betweenness as indicating control of resources or information. Importantly, however, although the degree and betweenness measures should indicate which nodes or criminals were the most central, targeting these criminals did not lead to a disruption of the criminal network and sometimes even led to the opposite effect, a stronger criminal network (Duijn et al., 2014; Firmani et al., 2014). This was because these centrality measures actually indicated the most vulnerable instead of the most powerful criminals: when one has many interactions with other criminals, this makes one easily traceable and thus more visible. As criminals do not want to be caught, this visibility is a weakness. Therefore, in this context, using centrality indices such as degree and betweenness did not give the expected information about the most influential or important nodes in the network.

Similarly, in psychological networks, such as symptom networks, it could also be that degree, closeness, and betweenness centrality do not play out as expected. For example, 
looking back at Figure 1, suppose we had a network with only nodes 3, 4, and 5. In this case node 4 would have the highest centrality indices on all three measures. A clinician might thus conclude that node or symptom 4 is the one to intervene on, based on the idea that the network structure is indicative of a causal structure. However, one way in which this partial correlation network structure can arise is through node 4 being a common effect of node 3 and 5 (i.e., node 5 and 3 both are causes of node 4). In this case intervening on node or symptom 4 would not change or disrupt any other symptoms in the network (Epskamp, 2017), and node 4 is in effect not a central node at all. More precisely, such a common effect node can be seen as an end point of a causal chain that cannot influence other nodes (Epskamp, 2017; Fried et al., 2018). Although temporal networks have directed edges, similar problems may arise in interpretability of centrality indices as the edges, for example, only represent unique, but not the shared variance of a VAR model (Bulteel et al., 2016).

\section{Ways Forward}

So far, we have discussed several issues with the most common centrality indices (i.e., degree, closeness, and betweenness) used in psychological network research. In general, it can be concluded that when using any centrality measure in social or psychological networks, its relevance and interpretability is highly reliant on the type of edge and process modelled. Therefore, it is not enough to state that one wants to measure how central a node is, but one has to make explicit what one means by a "central" or important node, and what assumptions the centrality measure of choice entails (Brandes, 2016; Schoch \& Brandes, 2016). Only in this way can it be transparent whether there is a match between the process under study and conclusions based on the centrality measure of interest (Borgatti, 2005; Borgatti \& Everett, 2006). Betweenness and closeness centrality seem especially poorly suited to most psychological networks, as they come with strong assumptions (such as that of the shortest 
paths) that may not hold (not only in psychological, but also in social and brain networks; Borgatti, 2005; Joyce, Laurienti, Burdette, \& Hayasaka, 2010). Furthermore, the edges in psychological networks indicate the (temporal) associations between nodes, and are as such informative and interpretable, but do not seem to correspond to a flow process. Further implicit assumptions of node distinctiveness and node exchangeability make these measures even less likely to be suited for psychological networks. Thus, with these problems in mind, the now most commonly used centrality indices do not seem ideal for studying psychological networks such as correlation, partial correlation, or VAR networks.

Where does this leave future research on centrality in psychological networks? We see three main ways forward: (a) using new centrality measures, (b) reconsidering the old measures of importance in the statistical models that underlie psychological networks, and (c) leaving the whole idea of centrality completely behind.

First, we could use other measures of centrality. The limitations of the three standard network measures have not gone unnoticed in the field of network science, and many alternative measures have been introduced over a substantial period of time (e.g., Agneessens, Borgatti, \& Everett, 2017; Ercsey-Ravasz, Lichtenwalter, Chawla, \& Toroczkai, 2012; Lawyer, 2015; Schoch, 2018; Yan, Zhai, \& Fan, 2013). For example, Schoch and colleagues recently introduced a new way of conceptualizing centrality that does not rely on the idea of shortest paths. Instead, this measure of centrality uses the notions of neighbor-inclusion and relative ranking instead of path lengths (Schoch, 2018; Schoch \& Brandes, 2016), potentially making it a better fit for psychological networks. In general, however, it is important to note that although new centrality measures have been introduced to address technical limitations such as the ability to include negative edges (e.g., Bonacich \& Lloyd, 2004), this does not as such solve the issues that were raised in this article. What is crucial is that centrality and other network measures should not just be technically appropriate, but should conceptualize 
centrality in a way that makes them meaningfully interpretable for psychological networks. Instead of simply applying new centrality measures to psychological networks, it would be a relevant endeavor to first put them under scrutiny, as we have now done with degree, closeness, and betweenness centrality, to determine whether they are suitable in a psychological network context.

A second way forward would be to use and improve measures of importance that have been specifically developed for the statistical models that we use for psychological networks. Centrality, or being the most influential or important variable, is not merely a question that has popped up in the network context, but has a rich history in psychological measurement. In that sense, psychological networks benefit from being based on existing statistical models that have seen an extensive history in developing ways to indicate relative variable importance. As Budescu (1993) states, since the development of regression and correlation analyses, people have sought ways to define importance of each variable through using, for instance, squared zero-order correlations, squared (semi-) partial correlation, dominance analyses, and many more (Budescu, 1993; Johnson \& LeBreton, 2004).

In the econometric literature on vector autoregressive models, importance of variables has also played a substantial role. In this context, impulse response functions are an often-used tool (for example see, Hamilton, 1994). This method can answer questions such as which variable has the largest impact on other variables in the network and how one could intervene to change a certain variable in the network, if the dynamics process that governs network evolution is adequately specified (Blaauw, van der Krieke, Emerencia, Aiello, \& de Jonge, 2017; Rosmalen, Wenting, Roest, de Jonge, \& Bos, 2012; Snippe et al., 2015). Interestingly, some of these measures have been introduced to the network literature already, such as the relative importance network (Bos et al., 2018; Bulteel et al., 2016; Robinaugh et al., 2014). One measure of relative importance is the proportional contribution that a predictor makes to 
$R^{2}$. When this is calculated for all the nodes with respect to one another, it results in a relative importance network. In these networks, strength centrality then has a clearer interpretation than in standard partial correlation networks, as both unique and shared variance is taken into account, and all edges are positive. However, in these networks too, betweenness and closeness centrality do not seem to be directly interpretable. In any case, instead of using or developing new centrality or importance measures, we could use and improve the measures that were made in the exact right context for that exact model (correlation or VAR) to answer questions on variable importance, and use the graphs only as an intuitive visualization of these results.

A third way forward would be to leave the idea of centrality indices behind completely. In centrality and relative importance measures, the focus is on identifying single variables to target for intervention, for instance, in a therapy setting. However, it is not clear if this is even possible at all. Variables such as symptoms are often intertwined, and even though therapists try to intervene on, for example, mood, it is likely that other things (e.g., going out more) will change at the same time. This phenomenon is also known as the fat-handed intervention, as things (thoughts and mood) are so interconnected that it is dubious whether interventions on a single variable, for example, worrying, are possible without changing the rest of the system (for more on this, see Eronen, 2018). Instead, the network approach can be seen as an incentive to move more towards more complex theories and models, belonging to the field of complex system theory (van de Leemput et al., 2014; Van Der Maas et al., 2006; Van der Maas \& Molenaar, 1992; Wichers, Groot, \& Psychosystems, 2016). Defining clinical disorders such as depression as a complex system network shows that shifting the focus away from single variables, to how the network emerges and behaves as a whole, might reveal more insights into the dynamics of psychopathology, leading to more fruitful therapy approaches (Borsboom, 2017; Cramer et al., 2016; Wichers, Wigman, \& Myin-Germeys, 2015). Thus, instead of trying to find the most central symptom, it might be better to study the dynamics as a whole, both to 
understand psychopathology and to know on which reciprocal associations or clusters of symptoms we should focus our interventions on.

Based on our careful dissection of the three commonly used centrality indices and their (often implicit) assumptions, we would recommend using these measures with considerable care in psychological networks. In particular betweenness and closeness centrality may be problematic in common applications, given that they have more complex assumptions, do not have a straightforward interpretation, and seem to be unstable in psychological networks. In general, it is important not to just choose a measure from the social network context, but first to make transparent what the (implicit) assumptions are for the measure, and why it is suited for the research question of interest. All in all, we hope to have helped to clarify what centrality indices do and do not measure in psychological networks.

\section{References}

Agneessens, F., Borgatti, S. P., \& Everett, M. G. (2017). Geodesic based centrality: Unifying the local and the global. Social Networks, 49, 12-26.

Anker, J. J., Forbes, M. K., Almquist, Z. W., Menk, J. S., Thuras, P., Unruh, A. S., \& Kushner, M. G. (2017). A network approach to modeling comorbid internalizing and alcohol use disorders. Journal of Abnormal Psychology, 126 , 325-339.

Anthonisse, J. M. (1971). The rush in a directed graph. Mathematische Besliskunde, No. BN 9/71. Amsterdam: Stichting Mathematisch Centrum.

American Psychiatric Association. (2013). Diagnostic and statistical manual of mental disorders (5th ed.). Washington, DC: Author. 
Bak, M., Drukker, M., Hasmi, L., \& van Os, J. (2016). An n= 1 clinical network analysis of symptoms and treatment in psychosis. PloS One, 11 , e0162811.

Barrat, A., Barthelemy, M., Pastor-Satorras, R., \& Vespignani, A. (2004). The architecture of complex weighted networks. Proceedings of the National Academy of Sciences, 101 , 3747-3752.

Bavelas, A. (1950). Communication patterns in task-oriented groups. The Journal of the Acoustical Society of America, 22 , 725-730.

Blaauw, F. J., van der Krieke, L., Emerencia, A. C., Aiello, M., \& de Jonge, P. (2017). Personalized advice for enhancing well-being using automated impulse response analysis—aira. Retrieved from https://arxiv.org/abs/1706.09268.

Bonacich, P., \& Lloyd, P. (2004). Calculating status with negative relations. Social networks, 26, 331-338.

Borgatti, S. P. (2005). Centrality and network flow. Social Networks, 27, 55-71. Borgatti, S. P., \& Everett, M. G. (2006). A graph-theoretic perspective on centrality. Social Networks, $28,466-484$.

Borsboom, D. (2008). Psychometric perspectives on diagnostic systems. Journal of Clinical Psychology, 64, 1089-1108.

Borsboom, D. (2017). A network theory of mental disorders. World Psychiatry, 16, 5-13.

Borsboom, D., \& Cramer, A. (2013). Network analysis: An integrative approach to the structure of psychopathology. Annual Review of Clinical Psychology, 9, 91-121.

Borsboom, D., Cramer, A., Schmittmann, V. D., Epskamp, S., \& Waldorp, L. J. (2011). The small world of psychopathology. PloS ONE, 6, e27407.

Borsboom, D., Fried, E., Epskamp, S., Waldorp, L., van Borkulo, C., van der Maas, H., \& Cramer, A. (2017). False alarm? A comprehensive reanalysis of "evidence that 
psychopathology symptom networks have limited replicability" by Forbes, Wright, Markon, and Krueger (2017). Journal of Abnormal Psychology, 126, 989-999.

Bos, F., Blaauw, F., Snippe, E., Van der Krieke, L., de Jonge, P., \& Wichers, M. (2018). Exploring the emotional dynamics of subclinically depressed individuals with and without anhedonia: an experience sampling study. Journal of Affective Disorders, 228, 186-193.

Boschloo, L., Schoevers, R. A., van Borkulo, C. D., Borsboom, D., \& Oldehinkel, A. J. (2016). The network structure of psychopathology in a community sample of preadolescents. Journal of Abnormal Psychology, 125, 599.

Boschloo, L., van Borkulo, C. D., Borsboom, D., \& Schoevers, R. A. (2016). A prospective study on how symptoms in a network predict the onset of depression. Psychotherapy and Psychosomatics, 85, 183-184.

Boschloo, L., van Borkulo, C. D., Rhemtulla, M., Keyes, K. M., Borsboom, D., \& Schoevers, R. A. (2015). The network structure of symptoms of the diagnostic and statistical manual of mental disorders. PloS ONE, 10, e 0137621.

Brandes, U. (2016). Network positions. Methodological Innovations, 9, 1-19.

Brandes, U., Borgatti, S. P., \& Freeman, L. C. (2016). Maintaining the duality of closeness and betweenness centrality. Social Networks, 44, 153-159.

Bringmann, L.F. (2016). Dynamical networks in psychology: More than a pretty picture? (Doctoral dissertation). Leuven: KU Leuven.

Bringmann, L. F., \& Eronen, M. I. (2018). Don't blame the model: Reconsidering the network approach to psychopathology. Psychological Review, 125, 606-615.

Bringmann, L. F., Lemmens, L. H., Huibers, M. J., Borsboom, D., \& Tuerlinckx, F. (2015).

Revealing the dynamic network structure of the Beck Depression Inventory-II. Psychological Medicine, 45, 747-757. 
Bringmann, L. F., Pe, M. L., Vissers, N., Ceulemans, E., Borsboom, D., Vanpaemel, W., . . . Kuppens, P. (2016). Assessing temporal emotion dynamics using networks. Assessment, 23, 425-435.

Bringmann, L. F., Vissers, N., Wichers, M., Geschwind, N., Kuppens, P., Peeters, F., . . . Tuerlinckx, F. (2013). A network approach to psychopathology: New insights into clinical longitudinal data. PLoS ONE, 8, e60188.

Budescu, D. V. (1993). Dominance analysis: a new approach to the problem of relative importance of predictors in multiple regression. Psychological Bulletin, 114, 542.

Bulteel, K., Tuerlinckx, F., Brose, A., \& Ceulemans, E. (2016). Using raw VAR regression coefficients to build networks can be misleading. Multivariate Behavioral Research, $51,330-344$.

Costantini, G., Epskamp, S., Borsboom, D., Perugini, M., Mõttus, R., Waldorp, L. J., \& Cramer, A. O. (2015). State of the aRt personality research: A tutorial on network analysis of personality data in R. Journal of Research in Personality, 54,13-29.

Costenbader, E. and Valente, T. W. (2003). The stability of centrality measures when networks are sampled. Social networks, 25, 283-307.

Cramer, A., van Borkulo, C., Giltay, E., van der Maas, H., Kendler, K., Scheffer, M., \& Borsboom, D. (2016). Major depression as a complex dynamical system. PLoS ONE, 11, e0167490.

Cramer, A., van der Sluis, S., Noordhof, A., Wichers, M., Geschwind, N., Aggen, S. H., . . . Borsboom, D. (2012). Dimensions of normal personality as networks in search of equilibrium: You can't like parties if you don’t like people. European Journal of Personality, 26, 414-431.

Cramer, A., Waldorp, L. J., van der Maas, H. L., \& Borsboom, D. (2010). Comorbidity: A network perspective. Behavioral and Brain Sciences, 33, 137-150. 
David, S. J., Marshall, A. J., Evanovich, E. K., \& Mumma, G. H. (2018). Intraindividual dynamic network analysis-implications for clinical assessment. Journal of Psychopathology and Behavioral Assessment, 40, 235-248.

Duijn, P. A., Kashirin, V., \& Sloot, P. M. (2014). The relative ineffectiveness of criminal network disruption. Scientific Reports, 4, 4238.

Epskamp, S. (2017). Network Psychometrics (Doctoral dissertation). Amsterdam: University of Amsterdam.

Epskamp, S., Borsboom, D., \& Fried, E. I. (2017). Estimating psychological networks and their accuracy: a tutorial paper. Behavior Research Methods, 50, 195-212.

Epskamp, S., Cramer, A., Waldorp, L. J., Schmittmann, V. D., \& Borsboom, D. (2012). Qgraph: Network visualizations of relationships in psychometric data. Journal of Statistical Software, 48, 1-18.

Epskamp, S., van Borkulo, C. D., van der Veen, D. C., Servaas, M. N., Isvoranu, A.-M., Riese, H., \& Cramer, A. O. (2018). Personalized network modeling in psychopathology: The importance of contemporaneous and temporal connections. Clinical Psychological Science, 6, 416-427.

Epskamp, S., Waldorp, L. J., Mõttus, R., \& Borsboom, D. (2018). The gaussian graphical model in cross-sectional and time-series data. Multivariate Behavioral Research, 53, 453-480.

Ercsey-Ravasz, M., Lichtenwalter, R. N., Chawla, N. V., \& Toroczkai, Z. (2012). Rangelimited centrality measures in complex networks. Physical Review E, 85, 066103.

Eronen, M. (2018) Causal Discovery and the Problem of Psychological Interventions. In: PSA 2018: The 26th Biennial Meeting of the Philosophy of Science Association (Seattle, WA; 1-4 November 2018).

URL: http://philsci-archive.pitt.edu/id/eprint/15224 
Firmani, D., Italiano, G. F., \& Laura, L. (2014). The (not so) critical nodes of criminal networks. In L. M. Aiello \& D. McFralan (Eds.), Social Informatics (pp. 87-96). New York, NY: Springer.

Forbes, M. K., Wright, A. G., Markon, K. E., \& Krueger, R. F. (2017). Further evidence that psychopathology networks have limited replicability and utility: Response to Borsboom et al. (2017) and Steinley et al. (2017). Journal of Abnormal Psychology, $126,1011-1016$.

Fortunato, S. (2010). Community detection in graphs. Physics reports, 486, 75-174.

Freeman, L. C. (1977). A set of measures of centrality based on betweenness. Sociometry, $40,35-41$.

Freeman, L. C. (1979). Centrality in social networks conceptual clarification. Social Networks, 1, 215-239.

Frewen, P. A., Allen, S. L., Lanius, R. A., \& Neufeld, R. W. (2012). Perceived causal relations novel methodology for assessing client attributions about causal associations between variables including symptoms and functional impairment. Assessment, 19, $480-493$.

Fried, E. I., \& Cramer, A. (2017). Moving forward: challenges and directions for psychopathological network theory and methodology. Perspectives on Psychological Science, 12, 999-1020.

Fried, E. I., Eidhof, M. B., Palic, S., Costantini, G., Huisman-van Dijk, H. M., Bockting, C. L., . . Karstoft, K.-I. (2018). Replicability and generalizability of posttraumatic stress disorder (PTSD) networks: a cross-cultural multisite study of PTSD symptoms in four trauma patient samples. Clinical Psychological Science, 6, 335-351.

Fried, E. I., Epskamp, S., Nesse, R. M., Tuerlinckx, F., \& Borsboom, D. (2016). What are 'good' depression symptoms? comparing the centrality of DSM and non-DSM 
symptoms of depression in a network analysis. Journal of Affective Disorders, 189, $314-320$.

Fried, E. I., \& Nesse, R. M. (2015). Depression sum-scores don't add up: why analyzing specific depression symptoms is essential. BMC Medicine, 13, 1-11.

Fried, E. I., van Borkulo, C. D., Cramer, A. O., Boschloo, L., Schoevers, R. A., \& Borsboom, D. (2017). Mental disorders as networks of problems: a review of recent insights. Social Psychiatry and Psychiatric Epidemiology, 52, 1-10.

Goldschmidt, A. B., Crosby, R. D., Cao, L., Moessner, M., Forbush, K. T., Accurso, E. C., \& Le Grange, D. (2018). Network analysis of pediatric eating disorder symptoms in a treatment-seeking, transdiagnostic sample. Journal of Abnormal Psychology, 127, $251-264$.

Gould, R. V. (1987). Measures of betweenness in non-symmetric networks. Social Networks, 9, 277-282.

Hamilton, J. D. (1994). Time series analysis. Princeton, NJ: Princeton University Press. Isvoranu, A.-M., Borsboom, D., van Os, J., \& Guloksuz, S. (2016). A network approach to environmental impact in psychotic disorder: brief theoretical framework. Schizophrenia Bulletin, 42, 870-873.

Isvoranu, A.-M., van Borkulo, C. D., Boyette, L.-L., Wigman, J. T., Vinkers, C. H., Borsboom, D., \& Investigators, G. (2016). A network approach to psychosis: pathways between childhood trauma and psychotic symptoms. Schizophrenia Bulletin, 43, 187-196.

Johnson, J. W., \& LeBreton, J. M. (2004). History and use of relative importance indices in organizational research. Organizational Research Methods, 7, 238-257.

Joyce, K. E., Laurienti, P. J., Burdette, J. H., \& Hayasaka, S. (2010). A new measure of centrality for brain networks. PLoS ONE, 5, e12200. 
Klippel, A., Viechtbauer, W., Reininghaus, U., Wigman, J., van Borkulo, C., MERGE, ... Wichers, M. (2017). The cascade of stress: a network approach to explore differential dynamics in populations varying in risk for psychosis. Schizophrenia Bulletin, 44, $328-337$.

Kolaczyk, E. D., \& Csárdi, G. (2014). Statistical analysis of network data with R. New York: Springer.

Kroeze, R., Van Veen, D., Servaas, M. N., Bastiaansen, J. A., Oude Voshaar, R., Borsboom, D., \& Riese, H. (2017). Personalized feedback on symptom dynamics of psychopathology: A proof-of-principle study. Journal of Person-Oriented Research, $3,1-10$

Laumann, E. O., Marsden, P. V., \& Prensky, D. (1989). The Boundary Specification Problem in Network Analysis. In L. C. Freeman, D. R. White, \& A. K. Romney (Eds.), Research methods in social network analysis (pp. 61-87). Piscataway, NJ: Transaction Publishers.

Lawyer, G. (2015). Understanding the influence of all nodes in a network. Scientific Reports, 5,8665 .

Leavitt, H. J. (1951). Some effects of certain communication patterns on group performance. The Journal of Abnormal and Social Psychology, 46, 38.

Levinson, C. A., Zerwas, S., Calebs, B., Forbush, K., Kordy, H., Watson, H., . . Bulik, C. M. (2017). The core symptoms of bulimia nervosa, anxiety, and depression: A network analysis. Journal of Abnormal Psychology, 126, 340-354.

Lord, F. M. (1953). On the statistical treatment of football numbers. American Psychologist, $8,750-751$.

Lü, L., Chen, D., Ren, X.-L., Zhang, Q.-M., Zhang, Y.-C., \& Zhou, T. (2016). Vital nodes identification in complex networks. Physics Reports, 650, 1-63. 
Marcus, D. K., Preszler, J., \& Zeigler-Hill, V. (2018). A network of dark personality traits: What lies at the heart of darkness? Journal of Research in Personality, 73, 56-62.

McNally, R. J., Robinaugh, D. J., Wu, G. W., Wang, L., Deserno, M. K., \& Borsboom, D. (2015). Mental disorders as causal systems: A network approach to posttraumatic stress disorder. Clinical Psychological Science, 3 , 836-849.

Moreno, J. (1934). Who shall survive? a new approach to the problem of human interrelations. Beacon, NY: Beacon House.

Moshier, S. J., Bovin, M. J., Gay, N. G., Wisco, B. E., Mitchell, K. S., Lee, D. J., . . Marx, B.P. (2018). Examination of posttraumatic stress disorder symptom networks using clinician-rated and patient-rated data. Journal of Abnormal Psychology, 127, 541547.

Newman, M. (2004). Analysis of weighted networks. Physical Review E, 70, 056131.

Newman, M. (2010). Networks: An introduction. Oxford: Oxford University Press.

Opsahl, T., Agneessens, F., \& Skvoretz, J. (2010). Node centrality in weighted networks: Generalizing degree and shortest paths. Social Networks, 32, 245-251.

Preszler, J., Marcus, D. K., Edens, J. F., \& McDermott, B. E. (2018). Network analysis of psychopathy in forensic patients. Journal of Abnormal Psychology, 127, 171-182.

Rhemtulla, M., Fried, E. I., Aggen, S. H., Tuerlinckx, F., Kendler, K. S., \& Borsboom, D. (2016). Network analysis of substance abuse and dependence symptoms. Drug and Alcohol Dependence, 161, 230-237.

Richetin, J., Preti, E., Costantini, G., \& De Panfilis, C. (2017). The centrality of affective instability and identity in borderline personality disorder: Evidence from network analysis. PloS ONE, 12, e0186695. 
Robinaugh, D. J., LeBlanc, N. J., Vuletich, H. A., \& McNally, R. J. (2014). Network analysis of persistent complex bereavement disorder in conjugally bereaved adults. Journal of Abnormal Psychology, 123, 510-522.

Robinaugh, D. J., Millner, A. J., \& McNally, R. J. (2016). Identifying highly influential nodes in the complicated grief network. Journal of Abnormal Psychology, 125 , $747-757$.

Rodebaugh, T. L., Tonge, N. A., Piccirillo, M. L., Fried, E., Horenstein, A., Morrison, A. S., . . . Heimberg, R.G. (2018). Does centrality in a cross-sectional network suggest intervention targets for social anxiety disorder? Journal of Consulting and Clinical Psychology, 86, 831-844.

Rosmalen, J. G., Wenting, A. M., Roest, A. M., de Jonge, P., \& Bos, E. H. (2012). Revealing causal heterogeneity using time series analysis of ambulatory assessments: Application to the association between depression and physical activity after myocardial infarction. Psychosomatic Medicine, 74, 377-386.

Ruzzano, L., Borsboom, D., \& Geurts, H. M. (2015). Repetitive behaviors in autism and obsessive-compulsive disorder: New perspectives from a network analysis. Journal of Autism and Developmental Disorders, 45, 192-202.

Sabidussi, G. (1966). The centrality index of a graph. Psychometrika, 31, 581-603.

Schoch, D. (2018). Centrality without indices: Partial rankings and rank probabilities in networks. Social Networks, 54, 50-60.

Schoch, D., \& Brandes, U. (2016). Re-conceptualizing centrality in social networks. European Journal of Applied Mathematics, 27, 971-985.

Scott, J. (2000). Social network analysis. London: Sage.

Shimbel, A. (1953). Structural parameters of communication networks. The Bulletin of Mathematical Biophysics, 15, 501-507. 
Smith, T. E., Lee, C. A., Martel, M. M., \& Axelrad, M. E. (2017). Odd symptom network during preschool. Journal of Abnormal Child Psychology, 45, 743-748.

Snijders, T. A. (2011). Statistical models for social networks. Annual Review of Sociology, 37, 131-153.

Snippe, E., Bos, E. H., van der Ploeg, K. M., Sanderman, R., Fleer, J., \& Schroevers, M. J. (2015). Time-series analysis of daily changes in mindfulness, repetitive thinking, and depressive symptoms during mindfulness-based treatment. Mindfulness, 6, 10531062.

Stephenson, K., \& Zelen, M. (1989). Rethinking centrality: Methods and examples. Social networks, 11, 1-37.

Tio, P., Epskamp, S., Noordhof, A., \& Borsboom, D. (2016). Mapping the manuals of madness: Comparing the ICD-10 and DSM-IV-TR using a network approach. International Journal of Methods in Psychiatric Research, 25, 267-276.

Valente, T. W., \& Foreman, R. K. (1998). Integration and radiality: Measuring the extent of an individual's connectedness and reachability in a network. Social Networks, 20, 89105.

van Borkulo, C. D., Borsboom, D., Epskamp, S., Blanken, T. F., Boschloo, L., Schoevers, R. A., \& Waldorp, L. J. (2014). A new method for constructing networks from binary data. Scientific Reports, 4, 5918.

van Borkulo, C. D., Boschloo, L., Borsboom, D., Penninx, B. W., Waldorp, L. J., \& Schoevers, R. A. (2015). Association of symptom network structure with the course of longitudinal depression. JAMA Psychiatry, 72, 1219-1226.

van de Leemput, I. A., Wichers, M., Cramer, A. O., Borsboom, D., Tuerlinckx, F., Kuppens, P., . . . others (2014). Critical slowing down as early warning for the onset and 
termination of depression. Proceedings of the National Academy of Sciences, 111, 87-92.

Van Der Maas, H. L., Dolan, C. V., Grasman, R. P., Wicherts, J. M., Huizenga, H. M., \& Raijmakers, M. E. (2006). A dynamical model of general intelligence: the positive manifold of intelligence by mutualism. Psychological review, 113, 842.

Van der Maas, H. L., \& Molenaar, P. C. (1992). Stagewise cognitive development: an application of catastrophe theory. Psychological Review, 99, 395.

van Roekel, E., Heininga, V. E., Vrijen, C., Snippe, E., \& Oldehinkel, A. (2018). Reciprocal associations between positive emotions and motivation in daily life: Network analyses in anhedonic individuals and healthy controls. Emotion, 19, 292-300.

Verschuere, B., van Ghesel Grothe, S., Waldorp, L., Watts, A. L., Lilienfeld, S. O., Edens, J. F., . . Noordhof, A. (2018). What features of psychopathy might be central? A network analysis of the Psychopathy Checklist-Revised (PCL-R) in three large samples. Journal of Abnormal Psychology, 127, 51-65.

Wasserman, S., \& Faust, K. (1994). Social network analysis: Methods and applications. Cambridge: Cambridge University Press.

Watts, D. J., \& Strogatz, S. H. (1998). Collective dynamics of 'small-world' networks. Nature, 393, 440-442.

White, D. R., \& Borgatti, S. P. (1994). Betweenness centrality measures for directed graphs. Social networks, 16, 335-346.

Wichers, M. (2014). The dynamic nature of depression: a new micro-level perspective of mental disorder that meets current challenges. Psychological Medicine, 44, 13491360. 
Wichers, M., Groot, P. C., \& Psychosystems. (2016). Critical slowing down as a personalized early warning signal for depression. Psychotherapy and Psychosomatics, 85, 114116.

Wichers, M., Wigman, J., \& Myin-Germeys, I. (2015). Micro-level affect dynamics in psychopathology viewed from complex dynamical system theory. Emotion Review, 7, 362-367.

Wigman, J., de Vos, S., Wichers, M., van Os, J., \& Bartels-Velthuis, A. A. (2016). A transdiagnostic network approach to psychosis. Schizophrenia Bulletin, 43, 122132.

Wigman, J., Van Os, J., Borsboom, D., Wardenaar, K., Epskamp, S., Klippel, A., . . . Wichers, M. (2015). Exploring the underlying structure of mental disorders: cross-diagnostic differences and similarities from a network perspective using both a top-down and a bottom-up approach. Psychological Medicine, 45, 2375-2387.

Yan, X., Zhai, L., \& Fan, W. (2013). C-index: A weighted network node centrality measure for collaboration competence. Journal of Informetrics, 7, 223-239. 
39

Running head: WHAT DO CENTRALITY MEASURES MEASURE?

Figure 1. The left figure is a hypothetical psychological network that can be thought of as a partial correlation network, in which the edges represent partial correlation and the nodes are typically symptoms or affect states. Red (or black) edges have negative values and blue (or grey) edges have positive values. The figure is inspired by Brandes, Borgatti, and Freeman (2016). The right figure is a plot of the centrality indices. Both figures are made with the R package qgraph. The figure shows that depending on the centrality index of interest different nodes can be labeled as most central. 


$$
5 y
$$

